\title{
ESTADO NUTRICIONAL DE IDOSOS INSTITUCIONALIZADOS: UMA REVISÃO DE LITERATURA
}

\section{NUTRITIONAL STATUS OF INSTITUTIONALIZED ELDERLY: A SYSTEMATIC REVIEW}

\author{
Jane Kátia Alves SILVÉRIO', Katherine Rios Almeida PEDREIRA², Nyvian Alexandre \\ $\mathrm{KUTZ}^{3}$, Marcia Maria Hernandes de Abreu de Oliveira SALGUEIRO ${ }^{4}$
}

\begin{abstract}
1 - Nutricionista. Centro Universitário Adventista de São Paulo, São Paulo, Brasil.
2 - Enfermeira. Mestranda em Promoção da Saúde pelo Centro Universitário Adventista de São Paulo, São Paulo, Brasil.

3 - Nutricionista. Mestranda em Nutrição Humana Aplicada pela Universidade de São Paulo, São Paulo, Brasil.

4 - Nutricionista. Professora da Graduação em Nutrição e do Mestrado em Promoção da Saúde do Centro Universitário Adventista de São Paulo, São Paulo, Brasil.
\end{abstract}

\section{RESUMO:}

O envelhecer traz em si muitas incertezas, principalmente em relação às condições de saúde e aos cuidados com a pessoa idosa. Diante disso, muitas famílias tendem cada vez mais a dividirem as responsabilidades do cuidado desses indivíduos com as Instituições de Longa Permanência para Idosos. O objetivo deste estudo foi verificar o estado nutricional de idosos institucionalizados avaliados pela Mini Avaliação Nutricional. Trata-se de uma revisão de literatura com evidências científicas. Foram utilizados artigos publicados e indexados nas bases de dados: Scientific Electronic Library Online (SciELO), Literatura Latino-Americana e do Caribe em Ciências da Saúde (Lilacs), National Center for Biotechnology Information NCBI (PUBMED) publicados nos últimos 5 anos. De acordo com a Mini Avaliação Nutricional destaca-se a alta prevalência de idosos institucionalizados em risco nutricional. A Mini Avaliação Nutricional é uma ferramenta útil e eficaz na detecção do estado nutricional de idosos e pode ser utilizada por profissionais da área da saúde, familiares e cuidadores de idosos. Os grupos de maior risco foram: idosos com deficiência cognitiva, capacidade física reduzida e de instituições da rede pública.

Palavras-chave: idoso; instituição de longa permanência para idosos; avaliação nutricional

\section{ABSTRACT:}

Aging brings with it many uncertainties, especially in relation to health conditions and care for the elderly. Faced with this, many families are increasingly inclined to share the care responsibilities of these individuals with Long-Term Care Facilities for the Elderly. The objective of this study was to verify the nutritional status of the institutionalized elderly evaluated by the Mini Nutritional Assessment. This is a literature review with scientific evidence. We used articles published and indexed in the databases: Scientific Electronic Library Online (SciELO), Latin American and Caribbean Literature in Health Sciences (Lilacs), National Center for Biotechnology Information NCBI (PUBMED) published in the last 5 years. According to the Mini Nutritional Assessment, the high prevalence of institutionalized elderly people at nutritional risk stands out. The Mini Nutritional Assessment is a useful and effective tool for detecting the nutritional status of the elderly and can be used by health professionals, family members and caregivers of the elderly. The groups at greatest risk were: elderly with cognitive deficiency, reduced physical capacity and public institutions.

Keywords: aged; homes for the aged; nutrition assessment 


\section{INTRODUÇÃO}

Segundo a Organização Mundial da Saúde, o aumento da longevidade é uma das maiores conquistas da humanidade, no entanto, constitui um dos maiores desafios mundiais. Estima-se que em 2025, haverá um total de aproximadamente dois bilhões de pessoas idosas, com 60 anos ou mais, em todo o mundo (WHO, 2005).

Este aumento está associado a mudanças severas nas estruturas e nos padrões familiares, assim como no trabalho e na migração. Com a urbanização acelerada e mudanças dos jovens para as grandes metrópoles à procura de trabalho, as famílias vêm se tornando cada vez menores. Nos últimos anos, houve um aumento significativo das mulheres no mercado de trabalho, o que reflete em menos pessoas disponíveis para cuidar dessa população idosa que vem crescendo a cada dia (WHO, 2005).

De acordo com Sousa et al. (2014), o processo do envelhecer traz em si muitas incertezas, principalmente no que se refere às condições de saúde, e os cuidados com a pessoa idosa. Com isso muitas famílias tendem cada vez mais a dividirem as responsabilidades do cuidado desses indivíduos com as Instituições de Longa Permanência para Idosos (ILPI).

As ILPI foram definidas pela Resolução RDC n²83/2005, da Agência Nacional de Vigilância Sanitária, como instituições governamentais ou não governamentais, de caráter residencial, destinada a domicílio coletivo de pessoas com idade igual ou superior a 60 anos, com ou sem suporte familiar, em condição de liberdade, dignidade e cidadania. A resolução define normas mínimas de funcionamento das ILPI, mas somente o Estatuto do Idoso produziu mudanças em relação à efetivação dos direitos dos idosos inseridos nesses locais, fornecendo instrumentos concretos para sua proteção. As ILPI estão sujeitas à fiscalização por parte do Ministério Público, dos Conselhos do Idoso e da Vigilância Sanitária, entre outros, ficando sujeitas, em caso de descumprimento de suas obrigações, a penalidades e à responsabilização civil e criminal de seus dirigentes ou prepostos, observado o devido processo legal (BRASIL, 2005). 
Nos países desenvolvidos e em desenvolvimento, têm aumentado os serviços de atenção aos idosos, principalmente aos que se encontram frágeis e debilitados pelo processo da idade, tanto em domicílio, quanto em ILPI. Esse tipo de serviço é uma forte tendência de atenção à saúde desses indivíduos (BRASIL, 2006).

O envelhecimento somado à diminuição da capacidade funcional, às alterações neurológicas, nutricionais e anatômicas e associados ao sedentarismo pode resultar em prejuízos à saúde e à qualidade de vida dos idosos. Com isso, têm-se por consequência a perda da autonomia, do autocuidado e em alguns casos da mobilidade, resultando em baixa autoestima e depressão (SPOSITO et al., 2013).

É sabido que o processo de envelhecimento pode comprometer o estado nutricional desses indivíduos, tanto em relação às patologias inerentes a idade avançada e/ou pelas alterações fisiológicas vividas nesse período. Estas alterações acontecem naturalmente no organismo do idoso e podem causar mudanças no estado nutricional, relacionadas com o ato da alimentação, dificuldade na mastigação pela perda dentária, diminuição da secreção salivar e secreções gástricas, o que caracteriza prejuízo na digestão, absorção e evacuação (WACHHOLZ, RODRIGUES, YAMANE, 2011).

Ao longo dos anos, os estudos têm mostrado um aumento significativo de idosos desnutridos, com prevalência entre 15 a 60\%, dependendo da instituição em que o idoso esteja internado, que pode ser em casa, asilo ou hospital e da técnica utilizada para determinar o estado nutricional (COLEMBERGUE, CONDE, 2011). Sendo assim, a avaliação periódica do estado nutricional para evitar e/ou detectar a desnutrição em idosos, tem um papel primordial nas ILPI. Os métodos mais utilizados envolvem avaliações dos parâmetros bioquímicos, antropométrico e a Mini Avaliação Nutricional (MNA) (OLIVEIRA et al., 2014). A MNA foi uma ferramenta desenvolvida e validada na cidade Toulouse, na França, em um estudo que comparou uma população de idosos frágeis a uma saudável em Albuquerque, Novo México, EUA (GUIGOZ, VELLAS, GARRY, 1994).

O teste consiste em 18 itens e quatro dimensões: medidas antropométricas, avaliação global, questionário dietético e uma avaliação subjetiva que permite classificar o estado nutricional em: normal (nutrição adequada), limite (em risco de desnutrição) ou 
desnutrição, a MNA é considerada um instrumento preciso para avaliar o estado nutricional de idosos (GUIGOZ, VELLAS, GARRY, 1994; FIDELIX, SANTANA, GOMES, 2013).

O presente estudo teve por objetivo verificar na literatura o estado nutricional de idosos institucionalizados avaliados pela MNA, e demonstrar que esta pode ser uma ferramenta útil para o diagnóstico nutricional.

\section{METODOLOGIA}

Trata-se de um estudo de revisão da literatura visando disponibilizar uma síntese das evidências relacionadas à temática, por meio da busca, análise e integração da informação selecionada. Foram utilizados artigos publicados e indexados nas bases de dados: Scientific Electronic Library Online - SciELO, Literatura Latino-Americana e do Caribe em Ciências da Saúde - Lilacs e National Center for Biotechnology Information NCBI (PUBMED). Os critérios de seleção dos artigos foram: artigos em português e inglês publicados nos últimos cinco anos (2010-2015), nos quais a MNA tivesse sido utilizada em idosos institucionalizados. Os descritores usados na busca estão expostos na Tabela 1.

Tabela 1. Descritores e bases de dados utilizados, na busca de artigos para revisão, São Paulo, 2015.

Português (SclELO e Lilacs)

Mini Avaliação Nutricional MNA

Idosos institucionalizados

Desnutrição em idosos

Uso da MAN em idosos

Avaliação nutricional em idosos
Inglês (PUBMED)

Mini Nutritional Assessment MNA

Aged institutionalized

Malnutrition in aged

Use the MNA in aged

Nutritional assessment in aged 
A primeira busca (por artigos em português) foi realizada nas bases de dados online SciELO e Lilacs, entre os meses de abril e maio de 2015. Foram encontrados 18 artigos e destes, 06 foram selecionados. A segunda busca (por artigos em inglês) foi realizada na base de dados PUBMED, nos meses de agosto e setembro de 2015, resultando em 371 artigos, dos quais 12 foram selecionados, totalizando 18 artigos, que foram lidos na íntegra, a fim de serem utilizados nesta revisão (Quadro1).

Quadro 1. Fluxograma da seleção dos artigos, São Paulo, 2015.

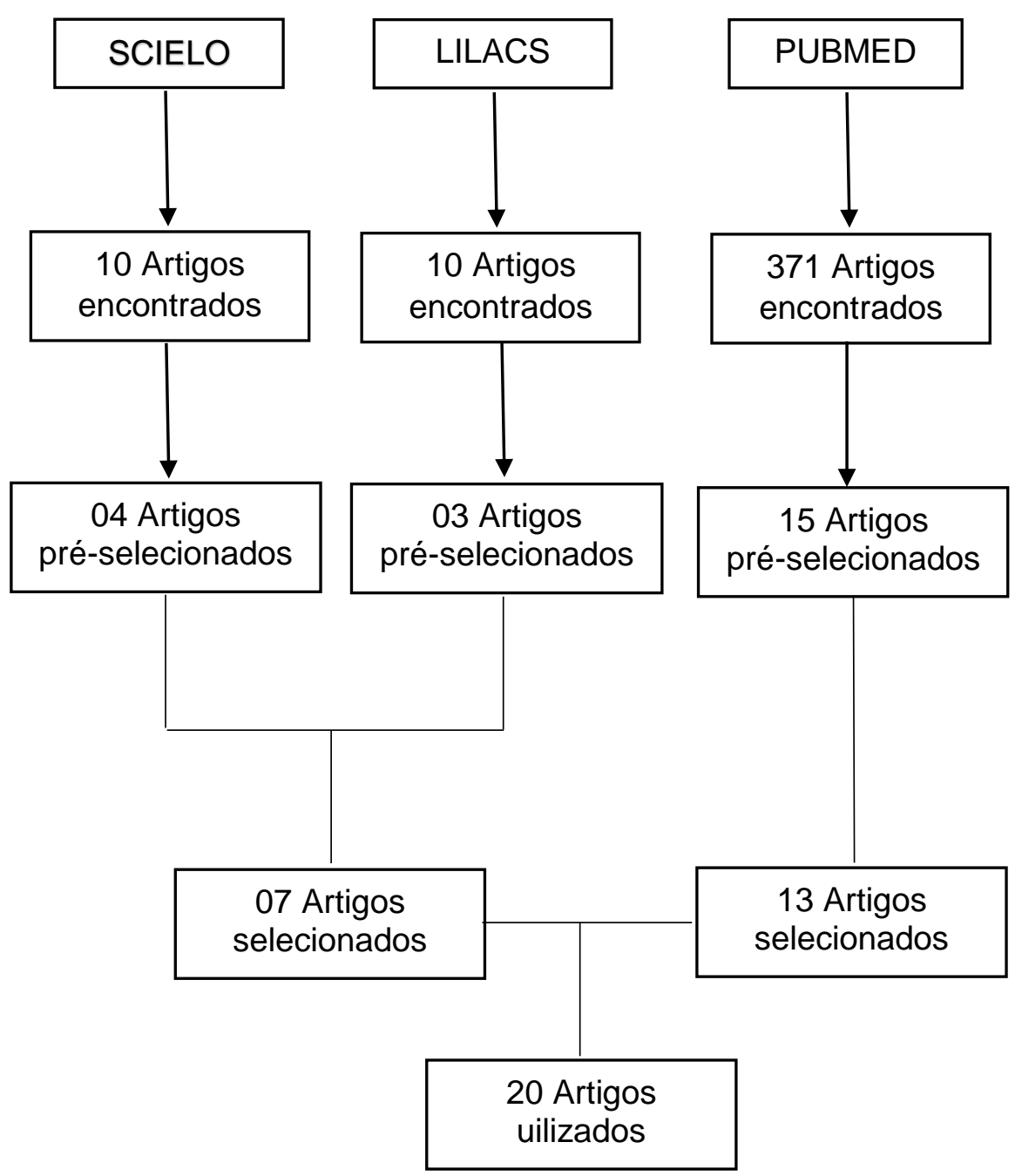




\section{RESULTADOS E DISCUSSÃO}

Nos 18 artigos selecionados para a revisão constatou-se que as publicações se concentraram entre 2011 e 2013. Desses estudos, o método quantitativo de pesquisa, de delineamento transversal foi preponderante, havendo apenas uma publicação que utilizou metodologia de revisão narrativa e dois estudos longitudinais. Dez estudos compararam a MNA com outros instrumentos de avaliação subjetiva. Quanto aos objetivos, observou-se que as pesquisas tenderam a determinar correlações e associações do estado nutricional e a idade e o número de doenças. A Tabela 2 apresenta o resumo dos artigos analisados.

Tabela 2. Resumo com os principais resultados dos artigos pesquisados, São Paulo, 2015.

\begin{tabular}{|c|c|c|c|}
\hline Autores/Ano & Objetivo do Estudo & Resultados & Conclusão \\
\hline $\begin{array}{l}\text { RAMBOUSKOVÁ, J. et al., } \\
2013\end{array}$ & $\begin{array}{c}\text { Avaliar o estado nutricional de } \\
\text { idosos institucionalizados em } \\
\text { Praga usando a MNA-reduzida e } \\
\text { outro indicadores nutricionais. }\end{array}$ & $\begin{array}{l}\text { Foram avaliadas } 65 \text { mulheres } \\
\text { das quais } 48 \% \text { tinham um bom } \\
\text { estado nutricional, } 41,3 \% \text { estavam } \\
\text { em risco de desnutrição e } 10,8 \% \\
\text { desnutridas. }\end{array}$ & $\begin{array}{l}\text { As mulheres institucionalizadas são } \\
\text { nutricionalmente mais vulneráveis. }\end{array}$ \\
\hline $\begin{array}{l}\text { SERRANO-URREA, R.; } \\
\text { GARCÍA-MESEGUER, M. J., } \\
2014\end{array}$ & $\begin{array}{c}\text { Estudar as relações entre } \\
\text { limitação funcional de idosos } \\
\text { institucionalizados, utilizando o } \\
\text { índice de Barthel (BI) e a MNA. }\end{array}$ & $\begin{array}{c}\text { Na amostra de } 895 \text { idosos } \\
\text { institucionalizados, o BI foi } \\
\text { correlacionado positivamente com } \\
\text { os seguintes itens: MNA, } \\
\text { mobilidade, auto alimentação, } \\
\text { exibição do status de integridade e } \\
\text { circunferência do braço. }\end{array}$ & $\begin{array}{l}\text { A MNA e o BI, estão positivamente } \\
\text { associados. As pontuações de BI } \\
\text { podem ajudar a determinar quem } \\
\text { pode estar em risco de má } \\
\text { nutrição. }\end{array}$ \\
\hline $\begin{array}{l}\text { MACHADO, R. S. P.; } \\
\text { COELHO, M. A. S. C., } 2011\end{array}$ & $\begin{array}{l}\text { Avaliar o estado nutricional de } \\
\text { idoso institucionalizados do Rio } \\
\text { de Janeiro, usando a ferramenta } \\
\text { MNA. }\end{array}$ & $\begin{array}{l}\text { Os } 344 \text { idosos que foram avaliados } \\
\text { pela MNA, apresentaram } 8,3 \% \text { de } \\
\text { desnutrição, } 55,6 \% \text { estavam em } \\
\text { risco de desnutrição e } 36,1 \% \\
\text { achavam-se eutróficos. }\end{array}$ & $\begin{array}{l}\text { O risco de desnutrição foi elevado } \\
\text { entre os idosos institucionalizados } \\
\text { da rede pública. }\end{array}$ \\
\hline TSAI, A. C. et al., 2010 & $\begin{array}{l}\text { Determinar se a adoção de } \\
\text { pontos de corte de IMC } \\
\text { específicos para a população } \\
\text { melhoraria a capacidade de } \\
\text { previsão da MNA-reduzida } \\
\text { e se a circunferência da } \\
\text { panturrilha (CP) poderia ser uma } \\
\text { alternativa aceitável ao IMC na } \\
\text { MNA-reduzida sem comprometer } \\
\text { sua capacidade preditiva. }\end{array}$ & $\begin{array}{l}\text { Estudo com } 369 \text { idosos de } 109 \\
\text { ILPI. Este estudo mostrou que o } \\
\text { uso dos três paramentos (a MNA- } \\
\text { reduzida, IMC para população } \\
\text { específica e a CP) foram } \\
\text { significativos para detectar a } \\
\text { desnutrição de idosos. }\end{array}$ & $\begin{array}{c}\text { A MNA pode ser um instrumento } \\
\text { útil e eficaz na detecção do estado } \\
\text { nutricional de idosos. A mesma } \\
\text { pode ser utilizada por profissionais } \\
\text { da área de saúde e cuidadores de } \\
\text { idosos. }\end{array}$ \\
\hline
\end{tabular}


VERONESE, N. et al., 2013 Correlacionar o IMC, pontuação da MNA, e os níveis de soro de albumina, com a taxa de mortalidade de 5 anos em idosos institucionalizados.

DE LUIS, D. A. et. al., 2011a Investigar e avaliar a desnutrição e a relação entre os diferentes marcadores antropométricos e bioquímicos, por meio da MNA.

OLIVEIRA, L. P. et al., 2014 Verificar as prevalências desnutrição em idosos de acordo com método, critérios e indicadores.

DE LUIS, D. A. et al., 2011b Investigar e avaliar, por meio de estudo multicêntrico, a prevalência de desnutrição bem como a relação entre dados antropométricos e marcadores bioquímicos com a MNA.

SOUSA, K. T. et al., 2014

WACHHOLZ, P. A.; RODRIGUES, S. C.; YAMANE, R., 2011

COLEMBERGUE, J. P.; CONDE, S. R.; 2011

Identificar a associação entre estado nutricional e dependência funcional dos idosos institucionalizados.

Analisar a correlação entre a percepção de qualidade de vida e o estado nutricional em homens idosos institucionalizados em uma unidade de longa permanência. idosos institucionalizados.
Neste estudo longitudinal, com

181 idosos institucionalizados, os resultados de 5 anos mostraram que a taxa de mortalidade foi de $63 \%$. O número de indivíduos falecidos foi 115 , todos tinham o IMC menor que $24,7 \mathrm{~kg} / \mathrm{m}^{2}$.

Os resultados demostraram alta prevalência de risco de desnutrição em ambos os sexos.

Entre os idosos com risco de desnutrição, não houve diferença entre os sexos.

Em 15 estudos $71,4 \%$ usaram a MNA como método para diagnóstico nutricional em idosos institucionalizados. Constatou-se que as prevalências de desnutrição variaram entre $6,3 \%$ a $81,7 \%$.

Dos 493 idosos estudados, de ambos os sexos residentes em ILPI, 245 (49,6\%) estavam em risco para desnutrição e 137

(22,5\%) estavam em desnutrição segundo a MNA. As mulheres apresentaram maior prevalência de desnutrição.

Estudos com 233 idosos institucionalizados. De acordo com a MNA, os idosos que tinham risco de desnutrição a faixa etária era de 81 anos.

Entretanto, os que não apresentaram riscos nutricionais a idade média era inferior que 76,5 .

Dos 35 idosos avaliados, verificou-se que $42,8 \%$ estavam com baixo peso. $34,3 \%$ eram eutróficos, 8,6\% estavam com sobrepeso e $14,3 \%$ achavam-se com obesidade.

Os 28 idosos institucionalizados foram avaliados por meio da MNA e, $10,7 \%$ estavam
O IMC é o melhor dos três parâmetros considerados como preditor de mortalidade e do estado nutricional de idosos institucionalizados a longo prazo.

Idosos com faixa etária entre 65-74 anos apresentam melhor estado nutricional que os mais velhos, enquanto que idosos com faixa etária >95 anos apresentam maior risco de desnutrição.

Existe uma grande variabilidade de prevalência de desnutrição em idosos institucionalizados, sendo a MNA o método mais utilizado para o diagnóstico.

Idosos institucionalizados tiveram alta prevalência de risco de desnutrição avaliados pela MNA.

O presente estudo demonstra claramente a necessidade de prevenção e reabilitação do baixo peso, bem como de ações que visem prevenir a dependência física dos idosos.

Dos idosos estudados nesta amostra, os melhores resultados foram para os que tinham menor

idade, eutróficos ou com sobrepeso, em relação aos indivíduos desnutridos ou com obesidade.

Houve uma baixa prevalência de idosos em risco de desnutrição e 
desnutridos, $25 \%$ com risco de desnutrição e $64,3 \%$ eutróficos.

MOREIRA, J. P. D.; MELO, Conhecer o perfil nutricional de P. T. H.; OLIVEIRA, A. M. C., idosas residentes em uma 2012 instituição geriátrica de Fortaleza (Ceará).

ONGAN, D.; RAKICIOĞLU, N., 2015

Avaliar o estado nutricional e ingestão alimentar de idosos institucionalizados na Turquia.

SERRANO-URREA, R.;

Avaliar a prevalência de GARCÍA-MESEGUER, M. J., 2013 desnutrição e risco de desnutrição em idosos que vivem em lares de idosos em província de Albacete (Espanha), usado a MNA.
Das 28 idosas avaliadas através da MNA, constatou-se que 4 delas $(14,3 \%)$ encontraram-se desnutridas, $2(7,1 \%)$ sob risco de desnutrição e 22 (78,6\%) eutróficas

De acordo com o MNA, 44,2\% apresentaram estado nutricional normal, $49,1 \%$ estavam em risco de desnutrição e 6,7\% apresentaram desnutrição.

Segundo o teste MAN aplicado em 895 idosos de ambos os sexos residentes em ILP, os resultados foram: $2,8 \% \quad(n=25)$ como desnutridos, $37,3 \%(n=334)$ em risco de desnutrição e 59,9\% $(n=536)$, eutróficos.

PEREIRA, A. S. M. L. et al., Avaliar o estado nutricional de 2014

LEE, L. C. et al., 2013

PAZ, R. C.; FAZZIO, D. M. Determinar o estado nutricional G.; SANTOS, A. L. B. et al., de idosos residentes em uma 2012 idosos vivendo em instituições na cidade de Salvador-BA e fatores associados.

Examinar a eficácia de um a triagem de rotina de acordo com necessidade de intervenção nutricional, e estratégia para melhorar o estado nutricional das pessoas que vivem em asilo.
Dos 359 idosos avaliados pela MNA, os resultados mostraram que $66,3 \%$ estavam desnutridos e em risco de desnutrição. Houve prevalência para os indivíduos do sexo masculino, $76,6 \%$ acharamse em risco de desnutrição e desnutridos.

Em estudo randomizado com 82 idosos de ambos os sexos, a MNA se destacou durante as 4 semanas do estudo, usando suplementação de $50 \mathrm{~g}$ de proteínas. Esta ferramenta possui sensibilidade (96\%) e especificidade (98\%) e é eficaz para avaliar idosos em várias fases da vida.

Nos 24 idosos avaliados por meio da MNA, houve prevalência de eutrofia em $66,7 \%(n=16)$ risco de desnutrição em $25 \%$ $(n=6)$ e desnutrição em 8,3\% $(n=2)$. desnutridos na população avaliada.

Entretanto a amostra foi restrita.

Conforme os resultados encontrados, pode-se inferir que as idosas avaliadas apresentaram um bom estado nutricional, já que a maioria se encontrou eutrófica.

\section{O estado nutricional de idosos} institucionalizados,

deve ser periodicamente reavaliado a fim de evitar à desnutrição.

Das perguntas da MNA as que melhor detectaram o estado nutricional dos idosos, foram as relacionadas com os dados antropométricos e autoavaliações.

Tem-se observado que muitos são os estudos usando a MNA como ferramenta para avaliação nutricional da população idosa.

Os resultados sugerem que uma intervenção nutricional seja realizada periodicamente, pois a população idosa vem aumentado mundialmente. E como consequência há um aumento na institucionalização. A MNA constitui uma ferramenta simples que pode auxiliar na estratégia para melhorar o estado nutricional desses indivíduos, reduzindo custos na saúde e mortalidade.

Neste estudo houve prevalência de desnutrição nos idosos segundo a classificação do IMC. O IMC teve forte correlação com a mortalidade e a morbidade. 
Houve uma alta prevalência de desnutrição em ambos os sexos, de acordo com avaliação da

MNA: $26 \%$ de mulheres e $16,3 \%$

de homens classificados como

sendo desnutridos, $40,9 \%$ de

mulheres e $35 \%$ de homens

estavam em risco de
Este estudo confirma a necessidade de se executar rotineiramente a avaliação do estado nutricional de idosos.

desnutrição.

*MNA Mini avaliação nutricional, IMC* Índice de Massa Corporal, "BI Índice de Barthel, *MAC Circunferência média do braço, *CC Circunferência da panturrilha.

O principal achado desta revisão indica alta prevalência de risco nutricional entre idosos institucionalizados avaliados pela MNA. A idade foi inversamente proporcional à classificação do estado nutricional pela MNA, ou seja, quanto maior a idade, há um declínio no estado nutricional (DE LUIS, et. al., 2011a). Estudos apontam que as idosas institucionalizadas estão mais desnutridas que os idosos (DE LUIS, et. al., 2011b; SERRANO-URREA; GARCÍA-MESEGUER, 2013).

O perfil nutricional de idosos é influenciado por condições socioeconômicas e fatores culturais do país (PINHEIRO; TORRES, 2006). Com isso, torna-se complexo avaliar e comparar ILPI nos diversos Estados de um mesmo país e entre países. Mais estudos devem ser realizados a fim de avaliar outros aspectos que influenciem o estado nutricional de idosos institucionalizados.

Durante a realização deste estudo, observou-se algumas características não abordadas nas pesquisas, mas que podem influenciar negativamente o estado nutricional: o tipo de instituição (pública ou privada), o número de idosos assistidos por instituição e a localização da mesma (grandes centros ou interior).

De Luis et al. (2011a), estudaram 873 idosos institucionalizados de ambos os sexos e encontraram alta prevalência de risco de desnutrição, principalmente entre os idosos com mais de 95 anos. Também foi observado melhor estado nutricional entre os idosos mais jovens, avaliados pela MNA-reduzida.

Ongan e Rakicioğlu (2015), em seu trabalho realizado na Turquia constataram alta prevalência de risco nutricional em todas as faixas etárias dos pacientes institucionalizados avaliados pela MNA. No entanto, em um estudo feito por Sousa et al. (2014), com idosos residentes em uma ILPI em Uberlândia-MG, encontraram alta prevalecia de risco de desnutrição somente entre idosos com idade mais avançada, avaliados por meio da MNA.

A MNA foi desenvolvida por pesquisadores internacionais e é uma das poucas ferramentas de controle validadas para os idosos, simples e de rápida aplicação. Tem sido 
utilizada e validada em estudos internacionais, em hospitais, na comunidade e em ILPI, e estabelece uma correlação entre morbidade e mortalidade (GUIGOZ, VELLAS, GARRY, 1994; GUIGOZ, 2006).

No estudo realizado por Pereira et al. (2014), em 15 ILPI com 359 idosos de ambos os sexos avaliados pela MNA, na cidade de Salvador - BA, verificou-se alta prevalência de risco de desnutrição e desnutrição nestes pacientes. Na comparação entre os sexos, observou-se que a maioria dos homens apresentaram essas condições.

Entretanto, Rambousková et al. (2013), avaliaram o estado nutrcional de 659 mulheres e 156 homens e constataram alta prevalência de risco nutricional e desnutrição. O estudo revelou a vulnerabilidade à desnutrição, morbidade e dependência física, principalmente nos indivíduos do sexo feminino. De Luis et al. (2011b), estudaram 493 idosos institucionalizados de ambos os sexos, e os resultados apontaram para alta prevalência de desnutrição ou risco de desnutrição segundo a MNA, sendo as mulheres mais desnutridas que os homens.

No entanto, Lee et. al. (2013), em um estudo randomizado com 82 idosos verificaram que a MNA teve lugar de destaque na aferição do estado nutricional em ambos os sexos, durante 4 semanas de intervenção nutricional usando suplementação de $50 \mathrm{~g}$ de proteínas. Também afirmam que a MNA é uma ferramenta que possui sensibilidade (96\%) e especificidade (98\%) e é eficaz para avaliar idosos em várias fases da vida.

Paz, Fazzio, Santos (2012) utilizaram a MNA para determinar o estado nutricional de idosos residentes em uma instituição particular do Distrito Federal -DF. Os resultados foram preponderantes para eutrofia segundo a MNA. Já um estudo realizado por Machado e Coelho (2011), em uma ILPI da rede pública do Rio de Janeiro- RJ, usando a ferramenta MNA, os resultados mostraram alta prevalecia de risco nutricional entre os idosos institucionalizados.

Veronese et al. (2013), correlacionaram o IMC com as pontuações da MNA e os níveis de albumina em um estudo de cinco anos. O IMC foi considerado o melhor dos três parâmetros na predição de mortalidade e do estado nutricional de idosos. Os resultados demonstraram que a taxa de mortalidade aumenta a medida que o IMC diminui. SerranoUrrea e García-Meseguer (2014), estudaram as relações entre limitação funcional de idosos institucionalizados utilizando o índice de Barthel (BI) e a MNA. Os resultados demonstram correlação positiva entre o BI e MNA, mostrando que esses indicadores associados podem ser ferramentas úteis na determinação de risco de desnutrição nesses indivíduos. 
Diante do exposto, destaca-se a importância da detecção precoce de risco de desnutrição e desnutrição em idosos. A MNA gera subsídios para profissionais intervirem com o suporte nutricional adequado com o objetivo de prevenir o declínio do quadro de desnutrição energético-proteica do paciente, melhorando a resposta ao tratamento proposto (VELLAS et al., 2006).

A implementação de intervenções nutricionais precoces são efetivas e muitas vezes têm baixo custo, atendendo às necessidades atuais do Sistema Único de Saúde (SUS) que conta com recursos escassos frente a grande demanda por serviços de saúde (SECOLI et al., 2010).

De acordo com o Ministério da Saúde, o envelhecimento populacional é um fenômeno natural, irreversível e mundial e a população idosa brasileira tem crescido de forma rápida. Desde a década de 60, observam-se os processos de transição demográfica, epidemiológica e nutricional no país, que resultam em alterações nos padrões de ocorrência de enfermidades (BRASIL, 2010).

Segundo o Estatuto do Idoso, no artigo 15 é assegurada a atenção integral à saúde do idoso, por intermédio do SUS, garantindo-Ihe o acesso universal e igualitário, por meio de um conjunto articulado e contínuo de ações e serviços, para prevenção, promoção, proteção e recuperação da saúde, incluindo a atenção especial às doenças que afetam preferencialmente os idosos. No artigo primeiro, destaca que a manutenção da saúde será garantida por meio do atendimento geriátrico e gerontológico na rede de saúde que conta com ambulatórios, unidades geriátricas de referência, atendimento domiciliar, incluindo a internação para os idosos impossibilitados de se locomover em instituições públicas, filantrópicas e sem fins lucrativos, nos meios urbano e rural (BRASIL, 2013).

$\mathrm{Na}$ atenção à saúde dos idosos também se busca a reabilitação desses indivíduos com redução das sequelas decorrentes dos agravos de saúde (BRASIL, 2010).

Consta no Estatuto do Idoso de 2013, que é dever das ILPI zelar pelo bem-estar e cuidados com a saúde dos idosos institucionalizados, priorizando ações que visem a promoção da saúde e de prevenção de incapacidades, levando em conta os aspectos culturais, sociais religiosos e políticos destes indivíduos. Essas ações devem ser planejadas e avaliadas pelo profissional responsável, em consenso com outros profissionais de saúde, bem como a equipe de cuidadores, levando em conta a opinião do residente e de seus familiares (BRASIL, 2013).

A maioria dos estudos apontam que os riscos de desnutrição aumentam dramaticamen $\neg$ te entre os idosos institucionalizados e hospitalizados, havendo prevalência 
de desnutrição ainda maior em idosos com deficiência cognitiva e capacidade funcional reduzida (GUIGOZ, VELLAS, GARRY, 1994).

É sabido que idosos que estão desnutridos quando são hos $\neg$ pitalizados, tendem a permanecer mais tempo inter $\neg$ nados, apresentam maiores complicações e correm maiores riscos de morbidade e mortalidade em relação àqueles que se encontram eutróficos (DONINI et al., 2004).

A MNA pode ser usada periodicamente tanto em hospitais quanto em ILPI, assim como na comunidade e ambiente familiar por ser um instrumento simples com alta afinidade para detectar o estado nutricional em idoso com o propósito de prevenir a desnutrição (VELLAS et al., 2006).

De acordo com a III Conferência Nacional dos Direitos da Pessoa Idosa realizada em 2011, o envelhecimento digno é um compromisso de todos. No Brasil, a efetivação desses direitos depende do comprometimento coletivo para que o Estatuto do Idoso não fique apenas no papel, é necessário que haja adesão do documento pela sociedade civil. A Comissão Permanente de Direitos Humanos estabeleceu como meta nacional o seguimento da criação e do funcionamento das ILPI e das demais unidades de atendimento a esse grupo etário, o que possibilita a promoção do envelhecimento com dignidade (BRASIL, 2011).

É importante salientar que, os cuidados realizados com estes pacientes institucionalizados, sejam individualizados e devidamente registrados em prontuários, com o propósito de serem reavaliados periodicamente e ajustados de acordo com a necessidade de cada idoso, tendo em vista que a MNA é uma ferramenta útil para o diagnóstico e o acompanhamento nutricional. Estes cuidados envolvem desde à higiene, alimentação, conforto, terapias ocupacionais além de cuidados mais complexos tendo como o foco à prevenção, reabilitação e promoção da saúde destes indivíduos (BRASIL, 2013).

A eventual ausência da família, ou ruptura de vínculos ou mesmo escassez de recursos financeiros para arcar com as despesas de contratação de um profissional para cuidar do idoso, podem levar a busca por ILPI. Diante desta realidade, faz-se necessário a ampliação de políticas públicas que garantam o atendimento institucional desses indivíduos (BRASIL, 2013).

Por isso a integração das famílias e da sociedade ao cotidiano da atenção aos idosos deu espaço para as ILPI se tornarem parceiras na construção do diálogo aberto com as famílias e pacientes. Desse modo podendo diminuir o preconceito em relação à 
institucionalização e às famílias que a ela recorrem (CREUTZBERG, GONÇALVES, SOBOTTKA, 2008).

Apesar de um estigma negativo em relação às ILPI, por envolvimento em situações de maus tratos e opressão à pessoa idosa, o que deve ser considerado e investigado, entretanto, há muitas experiências positivas de locais tranquilos e acolhedores para idosos, que vêm se tornando cada vez mais frequentes, sugerindo uma alternativa à demanda de moradia e cuidados para estes pacientes (BRASIL, 2013).

\section{CONCLUSÃO}

Na literatura estudada destaca-se alta prevalência de idosos institucionalizados em risco nutricional seguido de desnutrição avaliados pela MNA, os grupos de maiores riscos são: idosos com deficiência cognitiva, capacidade física reduzida e de instituições da rede pública.

Esses achados mostram a vulnerabilidade dos idosos institucionalizados para risco nutricional, sugerindo a implementação precoce e sistemática de avaliação nutricional e ações preventivas. A MNA pode gerar subsídios para os profissionais de saúde no monitoramento de riscos nutricionais e direcionar recursos para a atenção nutricional visando a promoção da qualidade de vida e à autonomia desta população.

\section{REFERÊNCIAS}

BRASIL. Ministério da Saúde. Agência Nacional de Vigilância Sanitária (ANVISA) - RDC № 283, de setembro 2005. Dispõe do Regulamento Técnico para o Funcionamento das Instituições de Longa Permanência para Idosos. Brasília, 2005. Disponível em: <http://bvsms.saude.gov.br/bvs/saudelegis/anvisa/2005/res0283_26_09_2005.html>. Acesso em: 08 mai. 2016.

BRASIL. Ministério da Saúde. Secretaria de Atenção à Saúde. Departamento de Atenção Básica. Envelhecimento e saúde da pessoa idosa. Brasília, 2006.192 p. Disponível em: <http://189.28.128.100/dab/docs/publicacoes/cadernos_ab/abcad19.pdf>. Acesso em: 17 abr. 2016.

BRASIL. Ministério da Saúde. Secretaria de Atenção à Saúde: Departamento de Atenção 
Básica. Envelhecimento e saúde da pessoa idosa. Brasília, 2010. 192p.Disponível em: <http://189.28.128.100/dab/docs/publicacoes/cadernos_ab/abcad19.pdf>. Acesso em: 18 abr. 2016.

BRASIL. Secretaria de Direitos Humanos. Estatuto do Idoso. Dignidade humana como foco. Brasília; 2013. 254 p. Disponível em: <http://www.sdh.gov.br/assuntos/pessoaidosa/publicacoes/estatuto-do-idoso-dignidade-humana-como-foco>. Acesso em: 18 abr. 2016.

BRASIL. Secretaria de Direitos Humanos. Secretaria Nacional de Promoção dos Direitos Humanos. A 3를 Conferência Nacional dos Direitos da Pessoa Idosa. Brasília, 2011. Disponível em: <http://www.sdh.gov.br/sobre/participacao-social/conselho-nacional-dosdireitos-do-Idoso-CNDI/conferencias/3a-conferencia/5-deliberacoes-iii-cndpi-2011>. Acesso em 21 abr. 2016.

COlEMBERGUE, J. P.; CONDE, S. R. Uso da Miniavaliação Nutricional em idosos institucionalizados Scientia Medica, Porto Alegre, v. 21, n. 2, p. 59-63, 2011.

CREUTZBERG, M.; GONÇALVES, L. H. T.; SOBOTTKA, A. E. Instituição de longa permanência para idosos: a imagem que permanece. Texto Contexto Enferm., Florianópolis, v. 17, n. 2, p. 273-279, 2008.

DE LUIS, D. A. et al. Evaluation of the mini-nutritional assessment short-form (MNA-SF) among institutionalized older patients in Spain. Nutr. Hosp., Madrid, v. 26, n. 6, p.13501354. 2011a.

DE LUIS, D. A. et al. Nutritional status in a multicenter study among institutionalized patients in Spain. Eur. Rev. Med. Pharmacol. Sci., Roma, v. 15, n. 3, p. 259-265, 2011 b.

DONINI, L. M. et al. Nutritional Care in a Nursing Home in Italy. PLoS ONE, v. 8, n. 2, p. e55804, 2013.

DONINI, L. M. et al. Prediction of stature in the Italian elderly. J. Nutr. Health Aging., Paris, v. 8, n. 13 , p. $386-388,2004$.

FIDELIX, S. P. M.; SANTANA, A. F. F.; GOMES, J. R. Prevalence of hospital malnutrition in 
elderly. RASBRAN, São Paulo, ano 5, n. 1, p. 60-68, 2013.

GUIGOZ, Y.; VELLAS, B.; GARRY, P. J. Mini nutritional assessment: a practical assessment tool for grading the nutritional state of elderly patients. Facts Res. Gerontol., Amsterdam, v. 4, supl. 2, p.15-59, 1994.

LEE, L. C. et al. Need-based intervention is an effective strategy for improving the nutritional status of older people living in a nursing home: A randomized controlled trial. Int. J. Nurs. Stud., Oxford, v. 50, n. 12, p. 1580-1588, 2013.

MACHADO, R. S. P.; COELHO, M. A. S. C. Risk of malnutrition among brazilian institutionalized elderly: a study with the mini nutritional assessment (MNA) questionnaire. J. Nutr. Health Aging, Paris, v. 15, n. 7, p. 532-535, 2011.

MOREIRA, J. P. D.; MELO, P. T. H.; OLIVEIRA, A. M. C. Nutritional profile of elderly female residing in a geriatric institution. Brazil. J. Health Promot., Fortaleza, v. 25, n. 1, p. 52-58, 2012.

OLIVEIRA, L. P. et al. Prevalência de desnutrição em idosos institucionalizados: uma revisão crítica sistemática. J. Health Biol. Sci., Teresina, v. 2, n. 3, p. 136-142, 2014.

ONGAN, D.; RAKICIOĞLU, N. Nutritional status and dietary intake of institutionalized elderly in Turkey: A cross-sectional, multi-center, country representative study. Arch. Gerontol. Geriatr., Amsterdam, v. 61, n. 2, p.271-276, 2015.

PAZ, R. C.; FAZZIO, D. M. G.; SANTOS, A. L. B. Avaliação nutricional em idosos institucionalizados. Revisa, Goiás, v.1, n.1, p. 9-18, 2012.

PEREIRA, S. A. L. M. et al. Nutritional status of institutionalized elderly Brazilians: a study with the Mini Nutritional assessment. Nutr. Hosp., Madrid, v. 31, n. 3, p. 1198-1204, 2014.

RAMBOUSKOVÁ, J. et al. Nutritional status assessment of institutionalized elderly in prague: Czech Republic. Ann. Nutr. Metab., New York, v. 62, n. 3, p. 201-206, 2013.

SECOLI, S. R. et al. Avaliação de tecnologia em saúde. II. A análise de custo-efetividade. Arq. Gastroenterol., São Paulo, v. 47, n. 4, p. 329-333, 2010.

SERRANO-URREA, R.; GARCÍA-MESEGUER, M. J. Malnutrition in an Elderly Population 
without Cognitive Impairment Living in Nursing Homes in Spain: Study of Prevalence Using the Mini Nutritional Assessment Test. Gerontology, Basel, v. 59, n. 6, p. 490-498, 2013.

SERRANO-URREA, R.; GARCÍA-MESEGUER, M. J. Relationships between nutritional screening: functional impairment in institutionalized Spanish older people. Maturitas, Amsterdam, v. 78, n. 4, p. 323-328, 2014.

SOUSA, K. T. et al. Baixo peso e dependência funcional em idosos institucionalizados de Uberlândia (MG), Brasil. Ciênc. Saúde Colet., Rio de Janeiro, v. 19, n. 8, p. 3513-3520, 2014.

SPOSITO, G. et al. Satisfação com a vida e a funcionalidade em idosos atendidos em um ambulatório de geriatria. Ciênc. Saúde Colet., Rio de Janeiro, v. 18, n. 12, p. 3475-3482, 2013.

TSAI, A. C. et al. Population-Specific Short-Form Mini Nutritional Assessment with Body Mass Index or Calf Circumference Can Predict Risk of Malnutrition in Community-Living or Institutionalized Elderly People in Taiwan. J. Am. Diet. Assoc., Chicago, v. 110, n. 9, p.1328-1334, 2010.

TSAI, A. C.; LAI, M. C.; CHANG, T. L. Mid-arm and calf circumferences (MAC and CC) are better than body mass index (BMI) in predicting health status and mortality risk in institutionalized elderly Taiwanese. Arch. Gerontol. Geriatr., Amsterdam, v. 54, v. 3, p. 443-447, 2012.

VELLAS, B. et al. Overview of the MNA - Its history and challenges. J. Nutr. Health Aging., Paris, v. 10, n. 6, p. 456-463, 2006.

VERONESE, N. et al. Body Mass Index as a Predictor of All-Cause Mortality in Nursing Home Residents During a 5-Year Follow-up. J. Am. Med. Dir. Assoc., Hagerstown, v. 14. n. 1. p. 53-57, 2013.

WACHHOLZ, P. A.; RODRIGUES, S. C.; YAMANE, R. Estado nutricional e a qualidade de vida em homens idosos vivendo em instituição de longa permanência em Curitiba, PR. Rev. Bras. Geriatr. Gerontol., Rio de Janeiro, v. 14, n. 4, p. 625-635, 2011.

WORLD HEALTH ORGANIZATION - WHO. Envelhecimento ativo: uma política de saúde. Brasília, 2005. 60p. Disponível em: <http://prattein.com.br/home/images/stories/ Envelhecimento/envelhecimento_ativo.pdf>. Acesso em: 07 mai. 2016. 